\title{
Sexual health risk reduction interventions for people with severe mental illness: a systematic review
}

Abdullah Pandor ${ }^{*}$, Eva Kaltenthaler ${ }^{1}$, Agnes Higgins ${ }^{2}$, Karen Lorimer ${ }^{3}$, Shubulade Smith ${ }^{4}$, Kevan Wylie ${ }^{5}$ and Ruth Wong ${ }^{1}$

\begin{abstract}
Background: Despite variability in sexual activity among people with severe mental illness, high-risk sexual behavior (e.g. unprotected intercourse, multiple partners, sex trade and illicit drug use) is common. Sexual health risk reduction interventions (such as educational and behavioral interventions, motivational exercises, counselling and service delivery), developed and implemented for people with severe mental illness, may improve participants' knowledge, attitudes, beliefs behaviors or practices (including assertiveness skills) and could lead to a reduction in risky sexual behavior. This systematic review evaluates the effectiveness of sexual health risk reduction interventions for people with severe mental illness.

Methods: Thirteen electronic databases (including MEDLINE, EMBASE and PsycINFO) were searched to August 2014, and supplemented by hand-searching relevant articles and contacting experts. All controlled trials (randomized or non-randomized) comparing the effectiveness of sexual health risk reduction interventions with usual care for individuals living in the community with severe mental illness were included. Outcomes included a range of biological, behavioral and proxy endpoints. Narrative synthesis was used to combine the evidence.
\end{abstract}

Results: Thirteen controlled trials (all from the USA) were included. Although there was no clear and consistent evidence that interventions reduce the total number of sex partners or improved behavioral intentions in sexual risk behavior, positive effects were generally observed in condom use, condom protected intercourse and on measures of HIV knowledge, attitudes to condom use and sexual behaviors and practices. However, the robustness of these findings is low due to the large between study variability, small sample sizes and low-to-moderate quality of included studies.

Conclusions: There is insufficient evidence at present to fully support or reject the identified sexual health risk reduction interventions for people with severe mental illness. Given the serious consequences of high-risk sexual behaviors, there is an urgent need for well-designed UK based trials, as well as training and support for staff implementing sexual health risk reduction interventions.

Trial registration: PROSPERO CRD42013003674.

Keywords: Systematic review, Schizophrenia, Bipolar disorder, Sexual behavior, Sexually transmitted diseases, Sexuality

\section{Background}

Severe mental illness (SMI), such as schizophrenia and bipolar disorder [1], persist over time and can result in extensive disability leading to impairments in social and occupational functioning [2]. Schizophrenia is estimated to affect approximately 180,471 [3] to 220,000 [4] people

\footnotetext{
* Correspondence: a.pandor@sheffield.ac.uk

${ }^{1}$ Health Economics and Decision Science, ScHARR, University of Sheffield, Sheffield, UK

Full list of author information is available at the end of the article
}

in the UK and bipolar disorder approximately 136,440 [3] to 297,000 [5]. While some individuals have long periods during which they are well and are able to manage their illness, many individuals with SMI have difficulties in establishing stable social and sexual relationships [6]. Despite variability in sexual activity among people with SMI (for example, people with schizophrenia-spectrum disorder are less likely than those with other major psychiatric disorders to be sexually active) [2], high-risk sexual behavior (e.g. unprotected intercourse, multiple

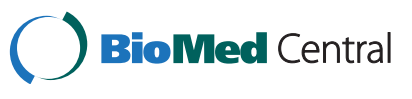

(C) 2015 Pandor et al.; licensee BioMed Central. This is an Open Access article distributed under the terms of the Creative Commons Attribution License (http://creativecommons.org/licenses/by/4.0), which permits unrestricted use, distribution, and reproduction in any medium, provided the original work is properly credited. The Creative Commons Public Domain Dedication waiver (http://creativecommons.org/publicdomain/zero/1.0/) applies to the data made available in this article, unless otherwise stated. 
partners, sex trade and illicit drug use) is common $[2,7,8]$ and rates of blood borne viruses, such as HIV and Hepatitis $C$, have been found to be higher among people with SMI [9] (including those who are homeless and/or have a substance misuse problem) $[10,11]$ than the general population. Risk behaviors for HIV among people with SMI can be influenced by substance use, childhood abuse, social relationships, and cognitivebehavioral factors $[2,12]$.

The US Centers for Disease Control and Prevention (CDC) list best- and good-level interventions for HIV prevention in the "Compendium of Evidence-Based HIV Behavioral Interventions", and continue to update this in light of new effectiveness evidence [13]. Behaviorally focused interventions have long been at the forefront of HIV prevention [14] and are characterized by their complexity and inclusion of multiple components. Behavioral strategies, such as those which attempt to delay onset of first intercourse, decrease the number of sexual partners or provide counselling and testing for HIV, can be focused at the level of individuals, couples, families and peer groups [15]. Interventions with, for example, an educational, behavioral, and/or counselling element could be developed and implemented for people with SMI, which may lead to improved participants' knowledge, attitudes, beliefs or behavioral practices (including assertiveness skills), which could lead to a reduction in risky sexual behavior [8]. However, this would require an understanding of the range of risk behaviors among those with SMI and an assessment of the effectiveness of interventions.

The aim of this research was to systematically review the evidence on the effectiveness of sexual health risk reduction interventions for people with SMI compared with usual care.

\section{Methods}

A systematic review was undertaken in accordance with the general principles recommended in the Preferred Reporting Items for Systematic Reviews and MetaAnalyses (PRISMA) statement [16].

\section{Data sources and searches}

Potentially relevant studies were identified through searches of thirteen electronic databases and research registers including MEDLINE (1948 to August 2014), EMBASE (1980 to August 2014), CINAHL (1982 to August 2014) and PsycINFO (1806 to August 2014). The search strategy used free text and thesaurus terms and combined synonyms relating to the condition (e.g. schizophrenia, psychotic disorders, bipolar disorder and severe mental illness) with terms for sexual health interventions (e.g. sexual behavior, sexually transmitted diseases and sexual health). A methodological filter aimed at restricting search results to controlled trials was used in the searches of MEDLINE, EMBASE, CINAHL and PsycINFO. Date and language restrictions were not used on any database (see online Additional file 1). Searches were supplemented by hand-searching relevant articles (including citation searching), systematic keyword searches of the World Wide Web and mental health organization websites and contacting experts in the field (including authors of three ongoing studies).

\section{Study selection}

Initially, all titles were examined by one reviewer. Citations that clearly did not meet the inclusion criteria were excluded i.e. non-human, unrelated to sexual health risk reduction interventions for people with SMI. Then, all abstracts and full text articles were examined independently by two reviewers. Any disagreements in the selection process were resolved through discussion. The systematic review included all controlled trials (randomized or non-randomized). Before and after studies without a concurrent control group were excluded because the absence of a control group to record concurrent changes over time means that changes due to the intervention or due to temporal trends, concurrent changes or a Hawthorne effect (a process in which subjects in a study change their behavior or performance in response to being observed) would be conflated. Such studies therefore represent very weak evidence of effectiveness $[17,18]$. Studies from developing countries were also excluded as it is difficult to generalize (e.g. transferability and acceptability) the characteristics of the effective interventions to developed countries. Eligible studies were those that included adult patients (aged over 18 years) with SMI living in the community. The term SMI usually refers to a severe and enduring mental illness associated with functional impairment that typically involves psychosis (losing touch with reality characterized by experiencing delusions and/or hallucinations) and commonly includes a diagnosis of schizophrenia, other psychoses and bipolar disorder [1]. In studies where there was a mixture of diagnostic groups, only those studies where the majority of participants (that is, more than $50 \%$ ) had psychotic diagnosis were included. We did not include studies where the sole diagnosis was major depression. Any health promotion intervention or combination of interventions (e.g. educational, behavioral, psychological, counselling etc. delivered at the individual, group and community level) intended to change the sexual knowledge, attitudes, beliefs, behaviors or practices of individuals and populations to improve their sexual health outcomes (by avoiding high risk sexual behavior through enhancing skills in, for example, decision making for safe sex and assertiveness with the aim of reducing adverse biological outcomes e.g. sexually 
transmitted diseases and HIV) were included. Interventions that focused on sexual dysfunction and sexual violence or sexual dysfunction attributable to the use of prescribed medications were excluded. The comparator was considered as standard usual care in the community and the main sexual health related outcomes included a range of biological (sexually transmitted infections including HIV, unintended pregnancy), behavioral (numbers of partners, use of contraception/condoms, uptake of screening or treatment services) and proxy (knowledge, attitudes and beliefs, barriers and facilitators, intentions, skills) endpoints.

Data abstraction and assessment of methodological quality Data relating to study design, methodological quality, and outcomes, were extracted by one reviewer into a standardized data extraction form and independently checked for accuracy by a second. Where multiple publications of the same study were identified, data were extracted and reported as a single study. The study quality characteristics were assessed according to (adapted) criteria based on those proposed by the Effective Public Health Practice Project - EPHPP [19]. This is a generic tool used to evaluate a variety of intervention study designs such as controlled trials and observational studies. This tool has been judged suitable to be used in systematic reviews of effectiveness [20] and has been reported to have content and construct validity $[19,21]$. Consideration of study quality included the following six criteria: [1] selection bias - the extent to which study participants were representative of the target population [2] study design; [3] control of confounders; [4] blinding - whether outcome assessors, intervention providers and participants were aware of the research question [5] data collection methods and [6] withdrawals and dropouts. The six domain-based criteria were each rated as strong, moderate or weak depending on the characteristics of each criterion reported in the included study (Table 1). An overall assessment of study quality was based on the following ratings: studies with at least four criteria rated as 'strong' and with no criteria rated as 'weak', were given an overall rating of 'strong'. Those studies receiving less than four 'strong' ratings and only one 'weak' rating were given an overall rating of 'moderate'. A rating of 'weak' was given if two or more criteria were rated as 'weak'. Additional study quality items included an assessment of intervention integrity, statistical analysis and generalizability to the UK. Any discrepancies in the data abstraction and quality assessment process were resolved through discussion to achieve agreement.

\section{Data synthesis and analysis}

A meta-analysis was not conducted on the data, as the studies were considered to be too heterogeneous with regards to the study designs, interventions and types of outcome data available. Therefore, as suggested by the guidance produced by the Cochrane Collaboration [22] and the Centre for Reviews and Dissemination (CRD) for undertaking systematic reviews [23,24], a narrative synthesis of included studies (grouped by outcome) was undertaken.

\section{Results}

\section{Trial flow}

Figure 1 summarizes the process of identifying and selecting relevant literature. Of the 2867 citations identified, 13 studies (representing 14 references) [25-38] met the inclusion criteria.

\section{Study and patient characteristics}

Full study details are presented in Table 2. All studies were published between 1996 and 2012 and were

Table 1 Quality assessment components and ratings for the EPHPP Instrument (Adapted with permission of the author) [19]

\begin{tabular}{|c|c|c|c|}
\hline Components & Strong & Moderate & Weak \\
\hline Selection Bias & $\begin{array}{l}\text { Very likely to be representative of } \\
\text { the target population and } \\
\text { participation rate }>80 \%\end{array}$ & $\begin{array}{l}\text { Somewhat likely to pre representative } \\
\text { of the target population and participation } \\
\text { rate between } 60 \text { to } 79 \%\end{array}$ & All other responses or not stated \\
\hline Study design & $\begin{array}{l}\text { Randomized controlled trial or } \\
\text { controlled clinical trial }\end{array}$ & $\begin{array}{l}\text { Cohort analytic, case control, cohort or an } \\
\text { interrupted time series }\end{array}$ & All other designs or design not stated \\
\hline Confounders & $\begin{array}{l}\text { Controlled for at least } 80 \% \text { of } \\
\text { confounders }\end{array}$ & $\begin{array}{l}\text { Controlled for at least } 60 \text { to } 79 \% \text { of } \\
\text { confounders }\end{array}$ & $\begin{array}{l}\text { Confounders not controlled for or not } \\
\text { stated }\end{array}$ \\
\hline Blinding & $\begin{array}{l}\text { Blinding of outcome assessors } \\
\text { and study participants to intervention } \\
\text { status and/or research question }\end{array}$ & $\begin{array}{l}\text { Blinding of either outcome assessors } \\
\text { or study participants }\end{array}$ & $\begin{array}{l}\text { Outcome assessors and study participants } \\
\text { are aware of intervention status and/or } \\
\text { research question }\end{array}$ \\
\hline $\begin{array}{l}\text { Data collection } \\
\text { methods }\end{array}$ & Tools shown to be valid and reliable & $\begin{array}{l}\text { Tools shown to be valid but reliability } \\
\text { not described }\end{array}$ & No evidenced of validity or reliability \\
\hline $\begin{array}{l}\text { Withdrawals and } \\
\text { dropouts }\end{array}$ & $\begin{array}{l}\text { Participants completing study } \\
\text { (or follow-up rate) }>80 \%\end{array}$ & $\begin{array}{l}\text { Participants completing study } \\
\text { (or follow-up rate) between } \\
60 \text { to } 79 \%\end{array}$ & $\begin{array}{l}\text { Participants completing study } \\
\text { (or follow-up rate) }<60 \% \text { or } \\
\text { withdrawals and dropouts not } \\
\text { described }\end{array}$ \\
\hline
\end{tabular}




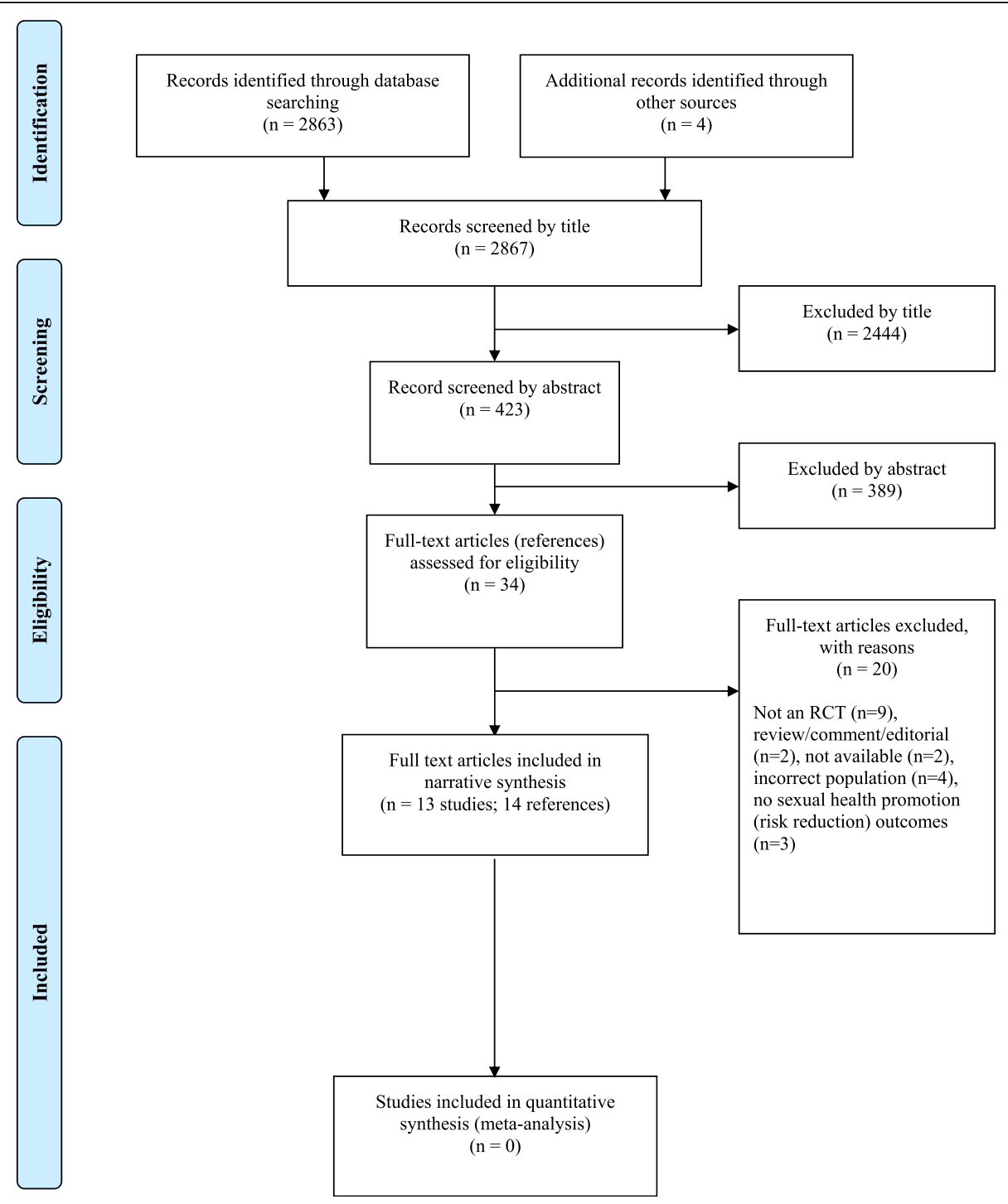

Figure 1 Study flow chart (adapted).

conducted in the USA (the majority of which were funded, at least in part, by the National Institute of Mental Health) [26,28-30,32,35-37]. Length of follow-up ranged from two weeks [31] to 18 months [36]. The content of the risk reduction interventions for improving sexual health varied between studies; however, most included HIV intervention programmes $[28-32,35]$ (that focused on providing education to enhance HIV knowledge and skills training and or cognitive behavioral therapy) to prevent or reduce the risk of HIV and skills development to negotiate and practice safe sex including developing condom use skills $[25,26,33,34,36,37]$. Although no explicit details were provided on the delivery method in each study, the most common deliverers were trained facilitators [28-30,32,34,37,38], mental health counsellors [26] or mental health professionals $[33,35,36]$. The duration of the intervention sessions ranged from four [30,31] to 15 sessions [36]. Standard usual care included educational sessions on HIV [25,27,32,33,37], money management [26,29,36], HIV and substance misuse education [28], waiting list or no treatment $[30,31,38]$, or health promotion covering a variety of topics $[34,35]$.

Study populations were recruited from different settings and included homeless shelters [25,27,33,36], outpatient psychiatric clinics $[26,28,30,32,35,37,38]$, residential facilities in a community setting [29], a drop-in socialization center [31] and a treatment programme for substance misusers [34]. Most studies included participants with a range of psychiatric diagnoses, which included schizophrenia, schizoaffective disorder, bipolar affective disorder and major depressive disorders. In one study [37], the psychiatric status and functioning of patients was not reported. For this 
Table 2 Summary of study characteristics of controlled trials

\begin{tabular}{|c|c|c|c|c|c|c|}
\hline Study, year & $\begin{array}{l}\text { Setting and } \\
\text { location }\end{array}$ & Population $^{\mathrm{a}}$ & Intervention & Control & Outcomes measured & Follow up \\
\hline \multirow{5}{*}{$\begin{array}{l}\text { Berkman et al. } \\
2006,[25,27]^{b}\end{array}$} & \multirow{5}{*}{$\begin{array}{l}\text { Homeless shelter, } \\
\text { New York, USA }\end{array}$} & Sample size: 92 & \multirow{3}{*}{$\begin{array}{l}\text { Social skills training approach with } \\
\text { cognitive-behavioral theory ( } 6 \text { sessions). } \\
\text { Details unclear on who delivered the } \\
\text { intervention. }\end{array}$} & \multirow{5}{*}{$\begin{array}{l}\text { Standard HIV ( } 1 \text { session) } \\
\text { education ( } n=42 \text {; of which } \\
23 \text { sexually active) }\end{array}$} & \multirow{5}{*}{$\begin{array}{l}\text { Unprotected anal, vaginal, } \\
\text { oral sex with casual partners } \\
\text { (women or men) as } \\
\text { measured by VEE score }\end{array}$} & \multirow[t]{5}{*}{6 months } \\
\hline & & Mean age: 38 years & & & & \\
\hline & & Male: $100 \%$ & & & & \\
\hline & & $\begin{array}{l}\text { Diagnosis: Schizophrenia or } \\
\text { schizoaffective disorder, 72\%; } \\
\text { bipolar disorder, 3\%; major } \\
\text { depressive disorder, } 10 \%\end{array}$ & \multirow{2}{*}{$\begin{array}{l}\text { Intervention included videos, role-playing } \\
\text { activities for development of skills, condom } \\
\text { use skills, negotiating safer sex, behavior } \\
\text { change, education on risks and problem } \\
\text { solving skills ( } n=50 \text {; of which } 33 \text { sexually } \\
\text { active) }\end{array}$} & & & \\
\hline & & Ethnicity: African-American, 65\% & & & & \\
\hline \multirow{5}{*}{$\begin{array}{l}\text { Berkman et al. } \\
\text { 2007,[26] }\end{array}$} & \multirow{5}{*}{$\begin{array}{l}\text { Outpatient } \\
\text { psychiatric clinics, } \\
\text { New York, USA }\end{array}$} & Sample size: 149 & \multirow{3}{*}{$\begin{array}{l}\text { Social skills training approach with } \\
\text { cognitive-behavioral theory ( } 10 \text { sessions } \\
\text { with boosters at } 3,6 \text { and } 9 \text { months) } \\
\text { delivered by substance abuse and/or } \\
\text { mental health counsellors. }\end{array}$} & \multirow{4}{*}{$\begin{array}{l}\text { Money-management with } \\
\text { matched treatment for } \\
\text { dosage and format of the } \\
\text { intervention group }(n=76)\end{array}$} & \multirow{5}{*}{$\begin{array}{l}\text { Unprotected anal, vaginal, } \\
\text { oral sex with casual partners } \\
\text { (women or men) as } \\
\text { measured by VEE score }\end{array}$} & \multirow[t]{5}{*}{12 months } \\
\hline & & Mean age: NR & & & & \\
\hline & & Male: $100 \%$ & & & & \\
\hline & & $\begin{array}{l}\text { Diagnosis: Schizophrenia, 49\%; } \\
\text { schizoaffective disorder, 22.8\%; } \\
\text { bipolar disorder, } 9.4 \% \text {; major } \\
\text { depressive disorder, 5.4\% }\end{array}$ & $\begin{array}{l}\text { Intervention included role-playing activities } \\
\text { for development of skills, condom use skills, } \\
\text { negotiating and practising safer sex (e.g. } \\
\text { ethics, goals, commitment), behavior } \\
\text { change, education on risks and problem } \\
\text { solving skills ( } \mathrm{n}=73 \text { ) }\end{array}$ & & & \\
\hline & & Ethnicity: African-American, 53.7\% & & & & \\
\hline \multirow{6}{*}{$\begin{array}{l}\text { Carey et al. } \\
2004,[28]^{\text {b }}\end{array}$} & \multirow{6}{*}{$\begin{array}{l}\text { Outpatient } \\
\text { psychiatric clinics, } \\
\text { New York, USA }\end{array}$} & Sample size: 408 & \multirow{4}{*}{$\begin{array}{l}\text { HIV risk reduction programme ( } 10 \\
\text { sessions) including enhancing knowledge } \\
\text { about HIV transmission, and prevention, } \\
\text { motivation for behavior change and } \\
\text { strengthening behavioral skills and } \\
\text { self-management training }(n=142)\end{array}$} & \multirow{6}{*}{$\begin{array}{l}\text { Standard care which included } \\
\text { HIV and substance use } \\
\text { education, if needed }(n=126)\end{array}$} & \multirow{6}{*}{$\begin{array}{l}\text { Frequency of unprotected } \\
\text { vaginal sex, total number of } \\
\text { sex partners, total number of } \\
\text { casual partners, number of } \\
\text { safer sex communications } \\
\text { before intercourse and } \\
\text { self-report of STIs }\end{array}$} & \multirow[t]{6}{*}{6 months } \\
\hline & & Mean age: 36.5 & & & & \\
\hline & & Male: $46 \%$ & & & & \\
\hline & & $\begin{array}{l}\text { Diagnosis: Schizophrenia, 18\%; } \\
\text { schizoaffective disorder, 15\%; } \\
\text { bipolar disorder, 19\%; major } \\
\text { depressive disorder, 49\% }\end{array}$ & & & & \\
\hline & & \multirow[t]{2}{*}{ Ethnicity: African-American, 21\% } & $\begin{array}{l}\text { Substance use reduction programme (10 } \\
\text { sessions) including enhancing } \\
\text { knowledge, motivation and skills to } \\
\text { reduce caffeine consumption, smoking, } \\
\text { and alcohol use ( } n=140)\end{array}$ & & & \\
\hline & & & $\begin{array}{l}\text { All interventions delivered by trained } \\
\text { clinical facilitators (with weekly } \\
\text { supervision from a licensed clinical } \\
\text { psychologist). }\end{array}$ & & & \\
\hline \multirow{3}{*}{$\begin{array}{l}\text { Collins et al. } \\
2011,[29]\end{array}$} & \multirow{3}{*}{$\begin{array}{l}\text { Urban community } \\
\text { setting, New York, } \\
\text { USA }\end{array}$} & Sample size: 79 & \multirow{3}{*}{$\begin{array}{l}\text { HIV prevention programme with social } \\
\text { cognitive theory ( } 10 \text { sessions) delivered } \\
\text { by trained facilitators (no further details } \\
\text { provided). }\end{array}$} & \multirow{3}{*}{$\begin{array}{l}\text { Money-management ( } 10 \\
\text { session workshop on } \\
\text { managing finances and last } \\
\text { through the month) }(n=40)\end{array}$} & \multirow{3}{*}{$\begin{array}{l}\text { Unprotected anal, vaginal, } \\
\text { oral sex with sexual partners } \\
\text { (casual, steady, exchange) as } \\
\text { measured by VEE score }\end{array}$} & \multirow[t]{3}{*}{6 months } \\
\hline & & Mean age: 42.3 & & & & \\
\hline & & Male: 0\% & & & & \\
\hline
\end{tabular}


Table 2 Summary of study characteristics of controlled trials (Continued)

\begin{tabular}{|c|c|c|c|c|c|c|}
\hline & & $\begin{array}{l}\text { Diagnosis: Schizophrenia, 50\%; } \\
\text { schizoaffective disorder/ } \\
\text { psychosis not specified, 14\%; } \\
\text { mood disorder with psychosis, } \\
\text { 13\%; mood disorder without } \\
\text { psychosis, 23\% }\end{array}$ & $\begin{array}{l}\text { Intervention focus was on self-efficacy and } \\
\text { skills training and included HIV/STI } \\
\text { awareness, risk prevention, self- } \\
\text { assertiveness, negotiating and practising } \\
\text { safer sex, condom use skills; problem } \\
\text { solving skills and commitment to } \\
\text { self-protection }(n=39)\end{array}$ & & & \\
\hline & & Ethnicity: Black, 61\% & & & & \\
\hline \multirow{5}{*}{$\begin{array}{l}\text { Kalichman et al. } \\
\text { 1995, [30] }\end{array}$} & \multirow{5}{*}{$\begin{array}{l}\text { Outpatient } \\
\text { psychiatric } \\
\text { community care, } \\
\text { Wisconsin, USA }\end{array}$} & Sample size: 52 & \multirow{3}{*}{$\begin{array}{l}\text { HIV prevention programme based on } \\
\text { behavioral skills training (4 sessions) } \\
\text { delivered by trained facilitators } \\
\text { experienced in HIV risk reduction } \\
\text { interventions. }\end{array}$} & \multirow{5}{*}{$\begin{array}{l}\text { Waiting list group (who later } \\
\text { received the intervention) } \\
(n=29)\end{array}$} & \multirow{5}{*}{$\begin{array}{l}\text { Knowledge, condom use, } \\
\text { behavior change } \\
\text { interventions }\end{array}$} & \multirow[t]{5}{*}{2 months } \\
\hline & & Mean age: 39.2 & & & & \\
\hline & & Male: $52 \%$ & & & & \\
\hline & & $\begin{array}{l}\text { Diagnosis: Schizophrenia, 62\%; } \\
\text { schizoaffective disorder, 23\%; major } \\
\text { affective disorder including bipolar, } \\
13 \%\end{array}$ & $\begin{array}{l}\text { Intervention included education on risk } \\
\text { reduction, sexual assertiveness, } \\
\text { negotiation skills (risk-related behavioral } \\
\text { self-management), condom use and } \\
\text { problem-solving skills }(n=23)\end{array}$ & & & \\
\hline & & Ethnicity: African-American, 19\% & & & & \\
\hline \multirow{5}{*}{$\begin{array}{l}\text { Katz et al. } \\
1996,[31]^{b}\end{array}$} & \multirow{5}{*}{$\begin{array}{l}\text { Outpatient } \\
\text { psychiatric centre, } \\
\text { California, USA }\end{array}$} & Sample size: 27 & \multirow{3}{*}{$\begin{array}{l}\text { AIDS education and risk reduction } \\
\text { training programme ( } 4 \text { sessions). Details } \\
\text { unclear on who delivered the } \\
\text { intervention. }\end{array}$} & \multirow[t]{5}{*}{ No treatment $(n=12)$} & \multirow{5}{*}{$\begin{array}{l}\text { Knowledge, behavior change } \\
\text { interventions }\end{array}$} & \multirow[t]{5}{*}{2 weeks } \\
\hline & & Mean age: NR & & & & \\
\hline & & $\begin{array}{l}\text { Male: NR but male female ratio } \\
2: 1\end{array}$ & & & & \\
\hline & & $\begin{array}{l}\text { Diagnosis: NR but majority of } \\
\text { patients diagnosed with } \\
\text { schizophrenia and bipolar } \\
\text { disorder }\end{array}$ & \multirow[t]{2}{*}{$\begin{array}{l}\text { Intervention included education about } \\
\text { HIV and AIDS, refusal skills training and } \\
\text { problem solving skills }(n=15)\end{array}$} & & & \\
\hline & & Ethnicity: NR & & & & \\
\hline \multirow{6}{*}{$\begin{array}{l}\text { Kelly et al. } \\
1997,[32]^{b}\end{array}$} & \multirow{6}{*}{$\begin{array}{l}\text { Outpatient } \\
\text { psychiatric care, } \\
\text { Wisconsin, USA }\end{array}$} & Sample size: 104 & \multirow{4}{*}{$\begin{array}{l}\text { Cognitive-behavioral therapy ( } 7 \text { sessions) } \\
\text { that focused on behavior changes to } \\
\text { reduce the risk of contracting HIV. } \\
\text { Interventions included education on risk } \\
\text { reduction, sexual assertiveness, } \\
\text { negotiation skills (risk-related behavioral } \\
\text { self-management), condom use and } \\
\text { problem-solving skills ( } n=34 \text { ) }\end{array}$} & \multirow{6}{*}{$\begin{array}{l}\text { A single } 60 \text { minute AIDS } \\
\text { education session }(n=28)\end{array}$} & \multirow{6}{*}{$\begin{array}{l}\text { AIDS risk behavior (knowledge } \\
\text { and safer-sex practices), and } \\
\text { condom use: barriers to } \\
\text { behavior change and } \\
\text { perceived risk reduction, } \\
\text { self-efficacy for use }\end{array}$} & \multirow[t]{6}{*}{3 months } \\
\hline & & Mean age: 33.7 & & & & \\
\hline & & Male: $47 \%$ & & & & \\
\hline & & $\begin{array}{l}\text { Diagnosis: Schizophrenia, 19\%; } \\
\text { mood disorder, 58\%; anxiety } \\
\text { disorder, 11\%; substance use or } \\
\text { personality disorder, } 11 \%\end{array}$ & & & & \\
\hline & & Ethnicity: African-American, 39\% & $\begin{array}{l}\text { Cognitive-behavioral therapy ( } 7 \text { sessions) } \\
\text { combined with advocacy training (to act } \\
\text { as a risk reduction advocate to their } \\
\text { friends and acquaintances) }(n=42)\end{array}$ & & & \\
\hline & & & $\begin{array}{l}\text { All interventions delivered by facilitators } \\
\text { (no further details provided) }\end{array}$ & & & \\
\hline
\end{tabular}


Table 2 Summary of study characteristics of controlled trials (Continued)

\begin{tabular}{|c|c|c|c|c|c|c|}
\hline \multirow{5}{*}{$\begin{array}{l}\text { Linn et al. 2003, } \\
\text { [33] }^{\mathrm{b}}\end{array}$} & \multirow{5}{*}{$\begin{array}{l}\text { Homeless shelter, } \\
\text { Nashville, USA }\end{array}$} & Sample size: 257 & \multirow{3}{*}{$\begin{array}{l}\text { Social skills training approach with } \\
\text { cognitive-behavioral theory ( } 6 \text { sessions) } \\
\text { delivered by HIV educators, a mental } \\
\text { health professional and a } \\
\text { 'paraprofessional'. }\end{array}$} & \multirow{5}{*}{$\begin{array}{l}\text { HIV and STI information (6 } \\
\text { sessions) and basic } \\
\text { instruction on condom use } \\
(n=127)\end{array}$} & \multirow{5}{*}{$\begin{array}{l}\text { Unprotected anal, vaginal, oral } \\
\text { sex with casual, occasional } \\
\text { and regular partners (women } \\
\text { or men) as measured by VEE } \\
\text { scorec }\end{array}$} & \multirow[t]{5}{*}{6 months } \\
\hline & & Mean age: NR & & & & \\
\hline & & Male: $100 \%$ & & & & \\
\hline & & $\begin{array}{l}\text { Diagnosis: Schizophrenia/ } \\
\text { schizoaffective disorder, 61\%; } \\
\text { major depression/ bipolar } \\
\text { disorder, 26\%; other, } 14 \%\end{array}$ & \multirow[t]{2}{*}{$\begin{array}{l}\text { Intervention included Sex, Games and } \\
\text { Videotapes with storytelling, competitive } \\
\text { games and acting scenes with true to } \\
\text { life scenarios }(n=130)\end{array}$} & & & \\
\hline & & Ethnicity: African-American, 54\% & & & & \\
\hline \multirow{5}{*}{$\begin{array}{l}\text { Malow et al. } \\
2012,[34]^{\mathrm{b}}\end{array}$} & \multirow{5}{*}{$\begin{array}{l}\text { Outpatient } \\
\text { psychiatric clinics, } \\
\text { Florida, USA }\end{array}$} & Sample size: 290 & \multirow{3}{*}{$\begin{array}{l}\text { Enhanced cognitive behavioral skill } \\
\text { building programme ( } 6 \text { sessions) } \\
\text { delivered by trained facilitators (no } \\
\text { further details provided). }\end{array}$} & \multirow{5}{*}{$\begin{array}{l}\text { Health promotion including } \\
\text { provision of information on } \\
\text { HIV, heart attacks, good food } \\
\text { habits, exercise, smoking and } \\
\text { stress }(n=126)\end{array}$} & \multirow{5}{*}{$\begin{array}{l}\text { HIV knowledge, perceived } \\
\text { susceptibility, AIDS related } \\
\text { anxiety, personal condom } \\
\text { attitudes, peer and partner } \\
\text { sexual attitudes, condom use } \\
\text { skills, sexual self-efficacy, total } \\
\text { number of unprotected vaginal } \\
\text { sex acts, proportion of } \\
\text { unprotected vaginal sex acts, } \\
\text { total number of sex partners. }\end{array}$} & \multirow[t]{5}{*}{6 months } \\
\hline & & Mean age: 39.6 & & & & \\
\hline & & Male: $45 \%$ & & & & \\
\hline & & $\begin{array}{l}\text { Diagnosis: schizophrenia, 15.7\%; } \\
\text { schizoaffective disorder, 8.4\%; } \\
\text { bipolar disorder, 9.6\%; major } \\
\text { depressive disorder, } 21.2 \%\end{array}$ & \multirow[t]{2}{*}{$\begin{array}{l}\text { Intervention included HIV education, } \\
\text { condom use, safe sex, high risk } \\
\text { situations, and communication skills } \\
(n=164)\end{array}$} & & & \\
\hline & & Ethnicity: African-American, 55\% & & & & \\
\hline \multirow[t]{5}{*}{ NIMH 2006,[37] $]^{b}$} & \multirow{5}{*}{$\begin{array}{l}\text { Outpatient mental } \\
\text { health clinics, New } \\
\text { York and Los } \\
\text { Angeles, USA }\end{array}$} & Sample size: 99 & \multirow{3}{*}{$\begin{array}{l}\text { Living in good health together } \\
\text { programme ( } 7 \text { sessions) delivered by } \\
\text { trained facilitators (no further details } \\
\text { provided). }\end{array}$} & \multirow{5}{*}{$\begin{array}{l}\text { A single AIDS education } \\
\text { session including video, } \\
\text { discussion, and referral } \\
\text { information ( } n=47 \text { ) }\end{array}$} & \multirow{5}{*}{$\begin{array}{l}\text { Number of partners; number } \\
\text { of risky sexual acts, proportion } \\
\text { of condom use; consistent } \\
\text { condom use }\end{array}$} & \multirow[t]{5}{*}{12 months } \\
\hline & & Mean age: NR & & & & \\
\hline & & Male: $100 \%$ & & & & \\
\hline & & $\begin{array}{l}\text { Diagnosis: NR but patients with } \\
\text { schizophrenia and bipolar } \\
\text { disorder were eligible }\end{array}$ & \multirow[t]{2}{*}{$\begin{array}{l}\text { Small group interventions covered } \\
\text { knowledge of HIV, personal triggers for } \\
\text { risk behavior, problem solving skills, } \\
\text { condom use, assertiveness, negotiation } \\
\text { strategies and relapse prevention } \\
(n=52)\end{array}$} & & & \\
\hline & & Ethnicity: African-American, $72.4 \%$ & & & & \\
\hline \multirow{4}{*}{$\begin{array}{l}\text { Otto-Salaj et al. } \\
2001,[35]^{b}\end{array}$} & \multirow{4}{*}{$\begin{array}{l}\text { Outpatient mental } \\
\text { health clinics, } \\
\text { Wisconsin, USA }\end{array}$} & Sample size: 189 & \multirow{3}{*}{$\begin{array}{l}\text { HIV prevention programme ( } 7 \text { sessions } \\
\text { with boosters at } 1 \text { and } 2 \text { months later) } \\
\text { delivered by trained mental health } \\
\text { facilitators. }\end{array}$} & \multirow{4}{*}{$\begin{array}{l}\text { Health promotion including } \\
\text { educational discussion and } \\
\text { skills building exercises } \\
\text { (focused on personal } \\
\text { relationships, stress, } \\
\text { nutritional health, cancer, } \\
\text { heart disease and general } \\
\text { sexual health) ( } \mathrm{n}=\mathrm{NR})\end{array}$} & \multirow{4}{*}{$\begin{array}{l}\text { HIV risk knowledge, attitudes } \\
\text { towards condom use; risk } \\
\text { reduction behavioral } \\
\text { intentions; frequency of } \\
\text { protected and unprotected } \\
\text { intercourse; intercourse } \\
\text { occasions protected by } \\
\text { condoms; number of } \\
\text { partners; }\end{array}$} & \multirow[t]{4}{*}{12 months } \\
\hline & & Mean age: 38.4 & & & & \\
\hline & & Male: $46 \%$ & & & & \\
\hline & & $\begin{array}{l}\text { Diagnosis: Schizophrenia, 35\%; } \\
\text { affective disorder, 34\%; } \\
\text { schizoaffective disorder, 18\%; } \\
\text { other, } 13 \%\end{array}$ & $\begin{array}{l}\text { Intervention included HIV risk reduction, } \\
\text { condom use, problem solving strategies, } \\
\text { discussion and role-play, negotiation and } \\
\text { assertiveness skills and behavior change } \\
\text { ( } n=\text { NR) }\end{array}$ & & & \\
\hline
\end{tabular}

Ethnicity: African-American, 51\% assertiveness skills and behavior change $(\mathrm{n}=\mathrm{NR})$ 
Table 2 Summary of study characteristics of controlled trials (Continued)

\begin{tabular}{|c|c|c|c|c|c|c|}
\hline \multirow{5}{*}{$\begin{array}{l}\text { Susser et al. 1998, } \\
{[36]}\end{array}$} & \multirow{5}{*}{$\begin{array}{l}\text { Homeless men, } \\
\text { New York, USA }\end{array}$} & Sample size: 59 (sexually active) & \multirow{3}{*}{$\begin{array}{l}\text { Social skills training approach with } \\
\text { cognitive-behavioral theory ( } 15 \text { sessions) } \\
\text { delivered by a mental health } \\
\text { professional and a 'paraprofessional'. }\end{array}$} & \multirow{5}{*}{$\begin{array}{l}\text { Health promotion ( } 2 \text { sessions) } \\
\text { including provision of } \\
\text { information on HIV, STI and } \\
\text { condom use }(n=26)\end{array}$} & \multirow{5}{*}{$\begin{array}{l}\text { Unprotected anal, vaginal, } \\
\text { oral sex with casual and } \\
\text { occasional partners (women } \\
\text { or men) as measured by VEE } \\
\text { score }^{c}\end{array}$} & \multirow[t]{5}{*}{18 months } \\
\hline & & Mean age: NR & & & & \\
\hline & & Male:100\% & & & & \\
\hline & & $\begin{array}{l}\text { Diagnosis: Schizophrenia/ } \\
\text { schizoaffective disorder, 61\%; } \\
\text { major depression/ bipolar } \\
\text { disorder, 27\%; other, } 12 \%\end{array}$ & \multirow[t]{2}{*}{$\begin{array}{l}\text { Intervention included Sex, Games and } \\
\text { Videotapes with storytelling, competitive } \\
\text { games and acting scenes with true to } \\
\text { life scenarios }(n=33)\end{array}$} & & & \\
\hline & & Ethnicity: African-American, 58\% & & & & \\
\hline \multirow{5}{*}{$\begin{array}{l}\text { Weinhardt et al. } \\
1998,[38]^{b}\end{array}$} & \multirow{5}{*}{$\begin{array}{l}\text { Outpatient } \\
\text { psychiatric care, } \\
\text { New York, USA }\end{array}$} & Sample size: 20 & \multirow{3}{*}{$\begin{array}{l}\text { Sexual assertiveness programme ( } 10 \\
\text { sessions) delivered by a facilitator (no } \\
\text { further details provided) }\end{array}$} & \multirow[t]{5}{*}{ No treatment $(n=11)$} & \multirow{5}{*}{$\begin{array}{l}\text { Sexual assertiveness, } \\
\text { knowledge, motivation, HIV } \\
\text { risk behavior }\end{array}$} & \multirow[t]{5}{*}{4 months } \\
\hline & & Mean age: 36 & & & & \\
\hline & & Male: $0 \%$ & & & & \\
\hline & & $\begin{array}{l}\text { Diagnosis: Schizophrenia } \\
\text { spectrum disorders, 50\%; bipolar } \\
\text { disorder, 30\%; major depressive } \\
\text { disorder, 20\% }\end{array}$ & \multirow[t]{2}{*}{$\begin{array}{l}\text { Intervention included HIV related } \\
\text { information and risk-behavior reduction, } \\
\text { skill acquisition and fluency building and } \\
\text { generalization of skills to actual } \\
\text { interactions }(n=9)\end{array}$} & & & \\
\hline & & Ethnicity: NR & & & & \\
\hline
\end{tabular}

NR, not reported; STI, sexually transmitted infections; VEE, vaginal episode equivalent.

${ }^{a}$ Characteristics at baseline.

${ }^{\mathrm{b}}$ Although all studies were described as RCTs by the study authors, this study did not report the method of randomization. According to the EPHPP quality assessment tool, this study would be categorized as a controlled clinical trial.

CThe VEE score is a sexual behavior risk index, It is calculated using the following formula: (number of un episodes). The VEE can be refined when data are extensive. For further details see Susser et al. [39]. 
study, it was assumed that people with mental health problems at high risk of HIV included some people with SMI. Sample sizes ranged from 20 [38] to 408 [28] patients, with mean participant age ranging from 33.7 [32] to 42.3 years [29] (data not reported in four studies) [31,33,36,37]. Five studies included males only [25-27,33,36,37], two women only $[29,38]$ and the remaining studies included both men and women (range from 45\% [34] to 52\% male) [30]. There was wide variation in the ethnicity of participants between the studies, with seven studies reporting the majority of participants to be of African-American origin [25-27,33-37]. Although four studies [28,32,35,38] provided no information on comorbidities, coexisting problems such as alcohol and drug dependence were common.

\section{Quality assessment}

A summary of the methodological quality of the included studies is presented in Figure 2. Generally, only two studies $[33,36]$ were considered as having very few methodological limitations. All studies, except one [37], selected participants that were 'somewhat likely' to be representative of the target population; however, most studies (54\%) did not report the number of individuals who were eligible to participate $[25,26,30,34,35,37]$ or reported very low numbers of eligible individuals who agreed to participate in the study [28]. Although all the studies were described as RCTs, only three studies reported the method of randomization $[26,29,36]$. In seven studies confounders were well controlled; [28,30,33-36,38] however, in the remaining studies $[25,26,29,31,32,37]$ no details on baseline compatibility were provided or if a variable was associated with the intervention or exposure and causally related to the outcome of interest. None of the studies were graded as 'strong' for blinding. Only five studies [26,28,33,34,36] blinded the outcome assessors and protected against detection bias. All studies failed to provide details on whether study participants were aware of the research question (reporting bias). Reliable and valid outcome measures were used in most $(77 \%)$ of the studies $[25,26,28-31,33,34,36,38]$. While three studies [30,32,38] failed to provide details of withdrawals and dropouts, the follow-up rate was $80 \%$ or greater in eight studies $[25,26,28,29,33,35-37]$. Intervention integrity (assurance that the intervention was delivered according to plan) is an important part of program delivery. Only five studies $[29,30,33,35,36]$ reported that more than $80 \%$ of participants received the allocated intervention and eight studies measured the consistency of the intervention [28-30,32,33,35-37], which was considered satisfactory. Contamination or co-intervention was unlikely in three studies [25,30,33], likely in one study [29] and not reported in nine studies [26,28,31,32,34-38]. No studies reported a sample-size calculation. Many of the studies had small sample sizes so it is likely they had inadequate statistical power to detect between group differences, even if they were present. The statistical analysis in most studies was appropriate and used intention-to-treat analysis. All the included studies were conducted in the USA, thus, making generalizability of the findings to the UK setting uncertain.

\section{Study results}

\section{Biological outcomes}

No studies evaluated the incidence of unintended pregnancies. Only one study [28] indicated that participation

\begin{tabular}{|c|c|c|c|c|c|c|c|}
\hline Author, year & Selection bias & Study design & Confounders & Blinding & Data collection & $\begin{array}{l}\text { Withdrawals and } \\
\text { drop outs }\end{array}$ & Overall rating \\
\hline Berkman et al. 2006(25;27) & Moderate & Strong & Weak & Moderate & Strong & Strong & Moderate \\
\hline Berkman et al. 2007(26) & Moderate & Strong & Weak & Moderate & Strong & Strong & Moderate \\
\hline Carey et al. 2004(28) & Weak & Strong & Strong & Moderate & Strong & Strong & Moderate \\
\hline Collins et al. 2011(29) & Moderate & Strong & Weak & Moderate & Strong & Strong & Moderate \\
\hline Kalichman et al. 1995(30) & Moderate & Strong & Strong & Moderate & Strong & Weak & Moderate \\
\hline Katz et al. 1996(31) & Moderate & Strong & Weak & Moderate & Strong & Weak & Weak \\
\hline Kelly et al. 1997(32) & Moderate & Strong & Weak & Moderate & Weak & Weak & Weak \\
\hline Linn et al. 2003(33) & Moderate & Strong & Strong & Moderate & Strong & Strong & Strong \\
\hline Malow et al. 2012(34) & Moderate & Strong & Strong & Moderate & Strong & Moderate & Moderate \\
\hline NIMH 2006(37) & Weak & Strong & Weak & Moderate & Weak & Strong & Weak \\
\hline Otto-Salaj et al. 2001(35) & Moderate & Strong & Strong & Moderate & Weak & Strong & Moderate \\
\hline Susser et al. 1998(36) & Moderate & Strong & Strong & Moderate & Strong & Strong & Strong \\
\hline Weinhardt et al. 1998(38) & Moderate & Strong & Strong & Moderate & Strong & Weak & Moderate \\
\hline
\end{tabular}


in a small-group, 10 session HIV risk-reduction and substance misuse reduction intervention significantly reduced the likelihood of new sexually transmitted infections (selfreported) over six months compared with usual care $(\mathrm{n}=$ 408) in men and women receiving outpatient psychiatric care.

\section{Behavioral outcomes}

No studies provided data on contraception use or uptake of screening or treatment services. Seven studies assessed the total number of sex partners in the past 30 days [32], six weeks $[33,36]$ or three months $[28,34,35,37]$. Two studies $[28,32]$ found a significant reduction in the number of sex partners $(n=512)$, whereas the remaining studies [33-37] found no significant effect compared with usual care $(\mathrm{n}=894)$.

Six studies reported data on unprotected intercourse. One study [32] observed significant reductions in rates of unprotected sex (anal, vaginal or oral) compared with usual care $(\mathrm{n}=104)$ in the past month. Five studies assessed sexual risk behavior using the vaginal episode equivalent (VEE) score (a weighted measure of transmission risk from unprotected oral, anal, or vaginal intercourse) [39]. Whilst two studies [33,36] found a significant improvement in the VEE scores $(n=316)$ over the previous six weeks, three studies observed no significant improvements either in the past six weeks [25] or three months $[26,29]$ compared with standard care $(n=$ 320 ). Five studies assessed condom use (in the past 30 days [30], six weeks [33,36] or three months) $[34,35,37]$ and one study evaluated condom use skills [34]. Two studies $[30,36]$ observed a significant increase in the number of times condoms were used in sexual intercourse $(n=111)$. Similarly, two studies $[33,35]$ found that more participants engaged (i.e. proportion of all encounters) in condomprotected intercourse (anal [33] or vaginal) [33,35] compared with usual care $(n=446)$; however, the effect was more pronounced in sex with women than men. One study [34] found a significant improvement in condom use skills (ability to properly enact steps in correctly placing a condom on a penis model), particularly in males, compared with usual care $(n=290)$. Although the majority of participants were of African-American origin in seven studies (range from 51\% [35] to 72.4\%) [37] only one study provided subgroup analysis data by ethnicity. This study [37] found that African-Americans in the intervention group reported significantly greater condom use (selfreported) than those in the control group; however, the intervention effect was not significant for Hispanic, Caucasian or other participants $(n=99)$.

\section{Proxy outcomes}

No studies evaluated barriers to, and facilitators of, sexual health promotion interventions for people with SMI.
Seven studies provided data on knowledge, attitudes and beliefs, intentions and skills, which are important mediators of effect. Although no improvement was found in one study [34] $(\mathrm{n}=290)$, six studies $[28,30-32,35,38](\mathrm{n}=800)$ observed an increase in HIV risk knowledge (in one of these studies this benefit was significant in men only, $\mathrm{n}=$ 189) [35]. Two studies [28,35] found significant improvements in attitudes for condom use $(n=597)$ when a HIV risk reduction programme was compared to usual care (in one of these studies this benefit was significant in women only but was not sustained beyond 12 months, $\mathrm{n}=189$ ) [35]. Behavioral intentions such as more frequent condom use or change in sexual risk behavior were improved in three studies $[28,30,34](\mathrm{n}=750)$. In contrast, no significant improvements were observed in two studies [35,38] $(\mathrm{n}=209)$. Compared with usual care, two studies $[28,38]$ observed a significant improvement in behavioral skills following sexual assertiveness training $(n=20)$ [38] or role-play simulations $(n=408)$ [28]. A detailed summary of the results for each included study is available in Kaltenthaler et al [40].

\section{Discussion}

People with SMI are a high-risk population for rates of blood borne viruses, including HIV and Hepatitis C compared with the general population. This systematic review examined the evidence on the effectiveness of sexual health risk reduction interventions for people with SMI compared with usual care and their applicability to the UK setting. We found no clear and consistent evidence across all studies for reductions in the total number of sex partners or improved behavioral intentions in sexual risk behavior. Although data were limited, positive effects were generally observed in condom use, condom protected intercourse and on measures of HIV knowledge, attitudes to condom use and sexual behaviors and practices. These results should be viewed cautiously as most positive findings were not consistently sustained in some long-term (12 months or more) follow-up studies $[35,36]$ and the low to moderate methodological quality of the majority of studies suggests the potential for bias in the study results. Despite the mixed findings of this systematic review, the results are broadly consistent with those of other reviews, but go beyond them because it includes data from eight additional studies $[25,26,28,29,31,33,34,37]$ and also includes a published search strategy (for reproducibility) and an assessment on the risk of bias, which were absent in existing reviews. Johnson-Masotti et al. [41] found limited success (positive effects were generally in studies with small sample sizes) of HIV prevention interventions at helping people with SMI reduce their HIV risk behavior. Whereas, Higgins et al. [8] found that people experiencing SMI who attended sexual health education programmes 
(which focused on topics such as HIV and other sexually transmitted diseases, negotiating safe sex and skill development in condom use and were facilitated in a sensitive and supportive manner) benefited and produced a reduction in sexual risk behavior as opposed to complete cessation. In addition, small-group interventions combining information giving, motivational exercises and skills acquisition were found to be effective in reducing sexual risk behavior and raise awareness of personal risk behavior. However, one-to-one teaching may also be preferred for some topics by some people.

The literature available was limited in quantity and heterogeneous with respect to study populations, content and duration of interventions and comparators and assessment of sexual health related outcomes. It is also worth drawing attention to other limitations. First, although an extensive literature search was conducted, it is possible that some relevant studies may have been missed. However, such omissions are likely to have been minimal, as the search included all identifiable publications in the grey literature (including contact with clinical experts in the field). Second, although studies from developing countries were excluded after the full text review stage, the core features of sexual health risk reduction interventions for people with SMI in this setting could be relevant and considered for adaptation in the UK; however, no relevant studies were found. Moreover, all identified and included studies were based in the USA, and included participants from diverse ethnic backgrounds (seven studies predominantly included participants of African-American origin) [25-27,33-37]. As a result, the transferability of this review to European settings such as the UK, which has a different multicultural society with different values and customs, is questionable. Third, most of the studies included in the systematic review included participants with major depression, which was not included in the definition of SMI used for this review (and that of the UK Department of Health strategy documents) [1,42]. Patients with major depression have different needs and risk and may respond differently to interventions than those with bipolar disorder or schizophrenia or indeed there may be differences between these latter diagnoses. However, it was not possible to differentiate results for patients with different diagnoses. In addition, the majority of studies recruited individuals from outpatient psychiatric centers where they were receiving treatment, thus the findings may not be generalizable to people with SMI who do not seek or receive specialist mental health care [43]. Fourth, sexual health risk reduction interventions were heterogeneous in terms of content, duration, how they were delivered and by whom; whereas usual care often included components of the intervention such as education. Consequently, uncertainties remain around the beneficial components of sexual health risk reduction programs. Fifth, most outcomes were based on the participants self-reported sexual risk behavior so this may be subject to possible response and recall bias. These and other limitations make it difficult to assess the true magnitude and direction of effect of the sexual health risk reduction interventions.

Although none of the studies reported that they had been designed to have adequate power to assess differences in subgroups, some reported differences in results between male and female participants. This issue needs to be explored further as there may be real differences between male and female responses to sexual health risk reduction interventions. In addition, there was a dearth of evidence on the relationship between education levels and outcomes, intervention acceptability from participants or those delivering the service, feasibility of the interventions and how much it would cost to implement. We would also note, in relation to HIV, that there are inherent limitations of the review methodology within a changing health domain may mean much of the work in this area is becoming dated [44]. Within the last year new biomedical approaches to HIV prevention, such as pre-exposure prophylaxis (PrEP) have become available in the USA, and in the UK it is proposed that home testing for HIV will be legalized in early 2014. New opportunities for intervention delivery, particularly technologically-based, exist, with feasibility, acceptability and effectiveness evaluated [45-47]. A recent systematic review of interactive computer-based interventions (delivered via the internet or other technology such as interactive television, mobile phone, CD-ROM and handheld computers) for sexual health promotion showed that computer-based interventions were effective tools for learning about sexual health and feasible in a variety of settings [48]. However, evidence that people with SMI are able to engage with technologically-based interventions (e.g. computer and mobile) to improve mental health services, is limited $[49,50]$. As a result, the current evidence base may now fail to adequately address the complexity and challenges of delivering sexual health improvement intervention modalities, particularly to patients with SMI. It remains important to note that new interventions have been developed from those conducted in the prehighly active antiretroviral era (HAART), and are rated as 'best evidence' by the CDC, such as Kelly's 'Popular Opinion Leader' intervention adapted for AfricanAmerican men who have sex with men [51].

Whilst there have been many initiatives in the UK to make health and social care more responsive and inclusive, the sexual health needs of individuals with psychosis appear to remain marginalized and neglected [52]. Despite variations in practice across the UK, the majority of mental health workers who are involved in the care of people with mental health problems (including 
SMI) in the UK do not provide sexual health promotion activities (including education) even though it is within the domain of their profession [53,54]. In contrast, in the Shetland Isles, Scotland, all mental health staff working with people with psychiatric illnesses receive sexual health training [55] although it is not clear exactly what this training includes and whether or not this varies with different types of staff.

Whilst people with SMI are able to engage in complex health interventions and benefit from them, several factors are relevant to implementing sexual health risk reduction interventions for people with SMI in the UK. These include consideration of who will deliver the interventions, where they will be delivered, whether sexual health risk reduction interventions could be integrated into the provision of current care and, if so how practitioners will be educated to deliver the interventions. A current on-going study [56] may provide some evidence on how physical co-morbidity in people with SMI could be addressed by community mental health nurses in the UK. The location of sexual health services for people with SMI and delivery of sexual health risk reduction interventions to groups or individuals will have resource implications and the potential to impact on effectiveness of the intervention. The long term impact of interventions, as well as the impact of 'timed booster education sessions' also needs to be taken into account as some of the included studies in the review showed a diminished impact at follow-up. Higgins et al. [8] suggests that sexual health education needs to be an ongoing process with sustained input, rather than a single intervention.

\section{Conclusions}

Despite the limitations to the review, this is the first comprehensive systematic review of sexual health risk reduction interventions for people with SMI. Previous reviews in this area have focused on HIV [2,57]. The large between study variability (especially in the populations, interventions, comparators, and reported outcomes) and mixed results, provide insufficient evidence at present to fully support or reject the identified sexual health risk reduction interventions for people with SMI. Given the lack of UK based studies, well designed trials of sexual health improvement interventions for people with SMI are warranted including training and support for staff implementing sexual health risk reduction interventions and an assessment of location and costs of proposed services. In addition, patient acceptability of proposed interventions also needs to be given careful consideration.

\section{Additional file}

Additional file 1: Literature search strategy, a MEDLINE example.
Competing interests

The authors declare that they have no competing interests.

\section{Author's contributions}

EK and AP were responsible for conception and design. RW developed the search strategy, undertook searches, and organized retrieval of papers. EK and AP were responsible for the acquisition, analysis and interpretation of data. $\mathrm{AH}, \mathrm{KL}$, SS and $\mathrm{KW}$ helped interpret data and provided a methodological, policy and clinical perspective on the data. AP wrote the drafts of the manuscript which were subsequently revised and approved by all authors. EK coordinated the review and is the guarantor.

\section{Acknowledgements}

We would like to thank Andrea Shippam, Project Administrator, ScHARR, for her help in the retrieval of papers and in preparing and formatting the paper.

\section{Funding}

This project was funded by the National Institute for Health Research Health Technology Assessment (NIHR HTA) Programme (project number: 12/74/12) as part of a review on 'The effectiveness of sexual health interventions for people with severe mental illness: a systematic review'. Visit the HTA programme website for more details: http://www.journalslibrary.nihr.ac.uk/ hta/volume-18/issue-1 (PROSPERO registration number: CRD42013003674). The views and opinions expressed therein are those of the authors and do not necessarily reflect those of the NHS, the NIHR, MRC, CCF, NETSCC, the NIHR HTA programme or the UK Department of Health.

\section{Author details}

${ }^{1}$ Health Economics and Decision Science, ScHARR, University of Sheffield, Sheffield, UK. ${ }^{2}$ School of Nursing and Midwifery, Trinity College Dublin, Dublin, Ireland. ${ }^{3}$ School of Health and Life Sciences, Glasgow Caledonian University, Glasgow, UK. ${ }^{4}$ Institute of Psychiatry at Kings College London, London, UK. ${ }^{5}$ Porterbrook Clinic, Sheffield, UK.

Received: 21 March 2014 Accepted: 20 January 2015

Published online: 12 February 2015

\section{References}

1. Government HM. No health without mental health: a cross-government mental health outcomes strategy for people of all ages. London: Department of Health; 2011.

2. Meade C, Sikkema K. HIV risk behavior among adults with severe mental illness: a systematic review. Clin Psychol Rev. 2005;25:433-57.

3. Kirkbride JB, Errazuriz A, Croudace TJ, Morgan C, Jackson D, McCrone P, et al. Systematic Review of the Incidence \& Prevalence of Schizophrenia and Other Psychoses in England, 1950-2009. Cambridge: University of Cambridge; 2011.

4. The Schizophrenia Commission. The abandoned illness: a report from the Schizophrenia Commission. London: Rethink Mental IIIness; 2012.

5. National Collaborating Centre for Mental Health. The management of bipolar disorder in adults, children and adolescents, in primary and secondary care. London: National Institute for Health and Clinical Excellence; 2006.

6. Robson D, Gray R. Serious mental illness and physical health problems: a discussion paper. Int J Nurs Stud. 2007:44:457-66.

7. Gray R, Brewin E, Noak J, Wyke-Joseph J, Sonik B. A review of the literature on HIV infection and schizophrenia: implications for research, policy and clinical practice. J Psychiatr Ment Health Nurs. 2002;9:405-9.

8. Higgins A, Barker P, Begley CM. Sexual health education for people with mental health problems: what can we learn from the literature? J Psychiatr \& Ment Health Nurs. 2006;13:687-97.

9. Rosenberg SD, Goodman LA, Osher FC, Swartz MS, Essock SM, Butterfield $\mathrm{MI}$, et al. Prevalence of HIV, hepatitis $B$, and hepatitis $C$ in people with severe mental illness. Am J Public Health. 2001;91:31-7.

10. Empfield M, Cournos F, Meyer I, McKinnon K, Horwath E, Silver M, et al. HIV seroprevalence among homeless patients admitted to a psychiatric inpatient unit. Am J Psychiatry. 1993;150:47-52.

11. Forney JC, Lombardo S, Toro PA. Diagnostic and other correlates of HIV risk behaviors in a probability sample of homeless adults. Psychiatr Serv. 2007;58:92-9. 
12. Marshall J, Farell M. Substance use and psychiatric comorbidity. Psychiatry. 2007;6:23-6.

13. Center for Disease Control and Prevention (CDC): Compendium of Evidence-Based HIV Behavioral Interventions (Electronic Version) [http://www.cdc.gov/hiv/prevention/research/compendium/index.html] Accessed January 16, 2014

14. Merson MH, O'Malley J, Serwadda D, Apisuk C. HIV prevention 1 - The history and challenge of HIV prevention. Lancet. 2008;372:475-88.

15. Coates TJ, Richter L, Caceres C. HIV Prevention 3 - Behavioural strategies to reduce HIV transmission: how to make them work better. Lancet. 2008;372:669-84

16. Moher D, Liberati A, Tetzlaff J, Altman DG. PRISMA Group. Preferred reporting items for systematic reviews and meta-analyses: the PRISMA statement. PLOS Med. 2009;6(7):e1000097. 10.1371/journal.pmed.1000097.

17. Armstrong R, Waters E, Jackson N. Guidelines for Systematic reviews of health promotion and public health interventions. Version 2. Australia: Melbourne University; 2007

18. Cook TD, Campbell DT. Quasi Experimentation. Design \& Analysis Issues for Field Settings. Houghton Mifflin Company: Boston, MA; 1979.

19. Thomas B, Ciliska D, Dobbins M, Micucci S. A process for systematically reviewing the literature: providing the research evidence for public health nursing interventions. Worldviews Evid Based Nurs. 2004;1:176-84.

20. Deeks J, Dinnes J, D'Amico R, Sowden AJ, Sakarovitch C, Song F, et al. Evaluating non-randomised intervention studies. Health Technol Assess. 2003;7:3-173.

21. Jackson N, Waters E. Criteria for the systematic review of health promotion and public health interventions. Health Promot Int. 2005;20:367-74.

22. Higgins JPT, Green S (Eds): Cochrane Handbook for Systematic Reviews of Interventions Version 5.1.0 [updated March 2011]. The Cochrane Collaboration; 2011. Available from http://handbook.cochrane.org/ webcite

23. Centre for Reviews and Dissemination (CRD). Systematic review: CRD's guidance for undertaking reviews in health care. York: University of York; 2009.

24. Khan K, Kunz R, Kleijnen J, Antes G. Systematic reviews to support evidencebased medicine. 2nd ed. London: Royal Society of Medicine; 2011.

25. Berkman A. Reducing sexual risk behaviors of men with severe mental illness. Psychiatr Serv. 2006;57:417.

26. Berkman A, Cerwonka E, Sohler N, Susser E. A randomized trial of a brief HIV risk reduction intervention for men with severe mental illness. Psychiatr Serv. 2006;57:407-9.

27. Berkman A, Pilowsky DJ, Zybert PA, Herman DB, Conover S, Lemelle S, et al. HIV prevention with severely mentally ill men: a randomised controlled trial. AIDS Care. 2007;19:579-88.

28. Carey M, Carey K, Maisto S, Gordon C, Schroder K, Vanable P. Reducing HIV-Risk Behavior Among Adults Receiving Outpatient Psychiatric Treatment: Results From a Randomized Controlled Trial. J Consult Clin Psychol. 2004;72:252-68.

29. Collins PY, von Unger H, Putnins S, Crawford N, Dutt R, Hoffer M. Adding the female condom to HIV prevention interventions for women with severe mental illness: a pilot test. Community Ment Health J. 2011;47:143-55.

30. Kalichman SC, Sikkema KJ, Kelly JA, Bulto M. Use of a brief behavioral skills intervention to prevent HIV infection among chronic mentally ill adults. Psychiatr Serv. 1995;46:275-80.

31. Katz RC, Westerman C, Beauchamp K, Clay C. Effects of AIDS counseling and risk reduction training on the chronic mentally ill. AIDS Educ Prev. 1996:8:457-63.

32. Kelly JA, McAuliffe TL, Sikkema KJ, Murphy DA, Somlai AM, Mulry G, et al. Reduction in risk behavior among adults with severe mental illness who learned to advocate for HIV prevention. Psychiatr Serv. 1997;48:1283-8.

33. Linn JG, Neff JA, Theriot R, Harris JL, Interrante J, Graham ME. Reaching impaired populations with HIV prevention programs: a clinical trial for homeless mentally ill African-American men. Cell Mol Biol. 2003;49:1167-75.

34. Malow RM, McMahon RC, Devieux J, Rosenberg R, Frankel A, Bryant V, et al. Cognitive behavioral HIV risk reduction in those receiving psychiatric treatment: a clinical trial. AIDS Behav. 2012;16:1192-202.

35. Otto-Salaj LL, Kelly JA, Stevenson LY, Hoffmann R, Kalichman SC. Outcomes of a randomized small-group HIV prevention intervention trial for people with serious mental illness. Community Ment Health J. 2001;37:123-44.

36. Susser E, Valencia E, Berkman A, Sohler N, Conover S, Torres J, et al. Human immunodeficiency virus sexual risk reduction in homeless men with mental illness. Arch Gen Psychiatry. 1998;55:266-72.
37. The National Institute of Mental Health (NIMH) Multisite HIV Prevention Trial Group. HIV prevention with persons with mental health problems. Psychol Health Med. 2006;11:142-54.

38. Weinhardt LS, Carey MP, Carey KB, Verdecias RN. Increasing assertiveness skills to reduce HIV risk among women living with a severe and persistent mental illness. J Consult Clin Psychol. 1998;66:680-4.

39. Susser E, Desvarieux M, Wittkowski KM. Reporting sexual risk behavior for HIV: a practical risk index and a method for improving risk indices. Am J Public Health. 1998;88:671-4.

40. Kaltenthaler E, Pandor A, Wong R. The effectiveness of sexual health interventions for people with severe mental illness: a systematic review. Health Technol Assess. 2014;18:1-74.

41. Johnson-Masotti AP, Weinhardt LS, Pinkerton SD, Otto-Salaj LL. Efficacy and cost-effectiveness of the first generation of HIV prevention interventions for people with severe and persistent mental illness. J Ment Health Policy Econ. 2003;6:23-35.

42. Department of Health. Choosing Health: Supporting the physical needs of people with severe mental illness - commissioning framework. London: Department of Health; 2006.

43. McAlpine DD, Mechanic D. Utilization of specialty mental health care among persons with severe mental illness: the roles of demographics, need, insurance, and risk. Health Serv Res. 2000;35:277-92.

44. Lorimer K, Kidd L, Lawrence M, McPherson K, Cayless S, Cornish F. Systematic review of reviews of behavioural HIV prevention interventions among men who have sex with men. AIDS Care. 2013;25:133-50.

45. Elford J, Hart G. If HIV prevention works, why are rates of high-risk sexual behavior increasing among MSM? AIDS Educ Prev. 2003;15:294-308.

46. Guse K, Levine D, Martins S, Lira A, Gaarde J, Westmorland W, et al. Interventions using New digital media to improve adolescent sexual health: a systematic review. J Adolesc Health. 2012;51:535-43.

47. Noar SA, Black HG, Pierce LB. Efficacy of computer technology-based HIV prevention interventions: a meta-analysis. AIDS. 2009;23:107-15.

48. Bailey JV, Murray E, Rait G, Mercer CH, Morris RW, Peacock R, et al. Interactive computer-based interventions for sexual health promotion. Cochrane Database Syst Rev. 2010;9:CD006483.

49. Ben-Zeev D, Davis KE, Kaiser S, Krzsos I, Drake RE. Mobile technologies among people with serious mental illness: opportunities for future services. Adm Policy Ment Health. 2013;40:340-3.

50. Mojtabai R, Corey-Lisle PK, Ip EH, Kopeykina I, Haeri S, Cohen LJ, et al. The patient assessment questionnaire: initial validation of a measure of treatment effectiveness for patients with schizophrenia and schizoaffective disorder. Psychiatry Res. 2012;200:857-66.

51. Kelly JA, Lawrence JSS, Diaz YE, Stevenson LY, Hauth AC, Brasfield TL, et al. HIV risk behavior reduction following intervention with Key opinion leaders of population - an experimental-analysis. Am J Public Health. 1991;81:168-71.

52. McCann E. The sexual and relationship needs of people who experience psychosis: quantitative findings of a UK study. J Psychiatr Ment Health Nurs. 2010;17:295-303.

53. Hughes E, Gray R. HIV prevention for people with serious mental illness: a survey of mental health workers' attitudes, knowledge and practice. J Clin Nurs. 2009;18:591-600.

54. Penna S, Sheehy K. Sex education and schizophrenia: should occupational therapists offer sex education to people with schizophrenia? Scand J Occup Ther. 2000;7:126-31.

55. Burtney E, Fullerton D. Sexual Health Improvement Interventions in Scotland. Mapping Exercise. NHS Health Scotland: Edinburgh; 2011.

56. White J, Gray R, Swift L, Barton G, Jones M. The serious mental illness health improvemnet profile [HIP]: study protocol for a cluster randomised controlled trial. BioMed Central. 2011;12:1-11.

57. Senn TE, Carey MP. HIV, STD, and sexual risk reduction for individuals with a severe mental illness: review of the intervention literature. Curr Psychiatry Rev. 2008:4:87-100. 